PROCEEDINGS OF THE

AMERICAN MATHEMATICAL SOCIETY

Volume 136, Number 12, December 2008, Pages 4111-4114

S 0002-9939(08)09565-8

Article electronically published on July 17, 2008

\title{
IMPROVEMENTS OF LOWER BOUNDS FOR THE LEAST COMMON MULTIPLE OF FINITE ARITHMETIC PROGRESSIONS
}

\author{
SHAOFANG HONG AND YUJUAN YANG
}

(Communicated by Wen-Ching Winnie Li)

\begin{abstract}
Let $u_{0}, r, \alpha$ and $n$ be positive integers such that $\left(u_{0}, r\right)=1$. Let $u_{k}=u_{0}+k r$ for $1 \leq k \leq n$. We prove that $L_{n}:=\operatorname{lcm}\left\{u_{0}, u_{1}, \cdots, u_{n}\right\} \geq$ $u_{0} r^{\alpha}(r+1)^{n}$ if $n>r^{\alpha}$. This improves the lower bound of $L_{n}$ obtained previously by Farhi, Hong and Feng.
\end{abstract}

\section{INTRODUCTION}

Arithmetic progression is an important topic in number theory. The renowned Dirichlet theorem tells us that any arithmetic progression with the first term and the common difference coprime contains infinitely many primes; see, for example, [1, [7] or [10. Recently, Green and Tao [5] proved a significant theorem saying that the set of primes contains arbitrarily long arithmetic progressions. On the other hand, Bachman and Kessler 22 and Myerson and Sander [1] investigated the divisibility properties of $\operatorname{lcm}\{1, \cdots, n\}$ (i.e., the least common multiple of all elements in the set $\{1, \ldots, n\}$ ) while Hong and Loewy $[9$ studied asymptotic behavior of eigenvalues of Smith matrices defined on arithmetic progressions.

The bounds for the least common multiple of finite arithmetic progressions have received lots of attention. Hanson [6] and Nair [12] derived the upper bound and lower bound of $\operatorname{lcm}\{1, \cdots, n\}$ respectively. Farhi [3, 4] obtained non-trivial lower bounds for the least common multiple of some finite arithmetic progressions. In what follows we always let $u_{0}, r$ and $n$ be positive integers such that $u_{0}$ and $r$ are coprime. Let $u_{k}=u_{0}+k r$ for $1 \leq k \leq n$ and define $L_{n}:=\operatorname{lcm}\left\{u_{0}, u_{1}, \cdots, u_{n}\right\}$. Farhi [3, 4] conjectured that for $n \geq 1$, we have $L_{n} \geq u_{0}(r+1)^{n}$. Note that this conjecture extends Nair's result [12] from the set $\{1, \cdots, n\}$ to a general arithmetic progression with $n$ terms. Hong and Feng [8] confirmed Farhi's conjecture. Meanwhile, Hong and Feng [8] obtained an improved lower bound under certain conditions. In fact, they proved that $L_{n} \geq u_{0} r(r+1)^{n}$ if $n>r$.

In this paper, our main interest is the lower bounds for the least common multiple of finite arithmetic progressions. We improve the above lower bound for $L_{n}$ if $n$ is large enough. We have the following result.

Received by the editors September 18, 2007.

2000 Mathematics Subject Classification. Primary 11A05.

Key words and phrases. Arithmetic progression, least common multiple, lower bound.

The first author was supported in part by the Program for New Century Excellent Talents in University, Grant No. NCET-06-0785.

(C)2008 American Mathematical Society Reverts to public domain 28 years from publication 
Theorem 1.1. Let $\alpha \geq 1$ be an integer. If $n>r^{\alpha}$, then $L_{n} \geq u_{0} r^{\alpha}(r+1)^{n}$.

The proof of Theorem 1.1 will be given in the third section. Throughout this paper, we let $[x]$ denote the integer part of a given real number $x$, as usual. We say that a real number $x$ is a multiple of a non-zero real number $y$ if the quotient $\frac{x}{y}$ is an integer.

\section{Preliminaries}

In the present section, we state some notations and known results which will be needed in the proof of Theorem 1.1. The following lemma was first stated in 3 . For an alternative proof, see [8].

Lemma 2.1 ([3], [4, 8]). For any positive integer $n, L_{n}$ is a multiple of $\frac{u_{0} u_{1} \cdots u_{n}}{n !}$.

For an integer $0 \leq k \leq n$, define $C_{n, k}:=\frac{u_{k} \cdots u_{n}}{(n-k) !}$ and $L_{n, k}:=\operatorname{lcm}\left\{u_{k}, \cdots, u_{n}\right\}$. Then $L_{n}=L_{n, 0}$. By Lemma 2.1 we have

$$
L_{n, k}=A_{n, k} \frac{u_{k} u_{k+1} \cdots u_{n}}{(n-k) !}=A_{n, k} C_{n, k},
$$

where $A_{n, k} \geq 1$ is an integer. Define

$$
k_{n}:=\max \left\{0,\left[\frac{n-u_{0}}{r+1}\right]+1\right\} .
$$

It is proved in [8] that for any $0 \leq k \leq n, C_{n, k} \leq C_{n, k_{n}}$. Evidently $L_{n}$ is a multiple of $L_{n, k}$ for all $0 \leq k \leq n$. Therefore for all $0 \leq k \leq n$, by (2.1) we have $L_{n} \geq L_{n, k} \geq C_{n, k}$. In particular, $L_{n} \geq C_{n, k_{n}}$. Thus we have the following result which also implies the truth of Farhi's conjecture.

Lemma $2.2([8])$. Let $C_{n, k}$ and $k_{n}$ be defined as above. Then $C_{n, k_{n}} \geq u_{0}(r+1)^{n}$. Consequently, we have $L_{n} \geq u_{0}(r+1)^{n}$.

\section{Proof of Theorem 1.1}

We are now in a position to prove Theorem 1.1.

Proof of Theorem 1.1. By [ [8], we know that Theorem 1.1 is true if $\alpha=1$ or $r=1$. It remains to consider the case where $\alpha \geq 2$ and $r \geq 2$.

In the following we let $\alpha \geq 2$ and $r \geq 2$. We first treat the special case where $\alpha=r=2, u_{0}=1$ and $n=5$. For this special case, we have

$$
L_{n}=\operatorname{lcm}\{1,3,5,7,9,11\}=3465 \text { and } u_{0} r^{\alpha}(r+1)^{n}=972 .
$$

Thus we have $L_{n} \geq u_{0} r^{\alpha}(r+1)^{n}$. That is, Theorem 1.1 holds for the special case.

Next, we deal with the remaining cases except for the above special case. We claim that $\alpha r \leq n-k_{n}$ except for the special case. Notice that $r^{\alpha} \geq \alpha r$ since $\alpha \geq 2$ and $r \geq 2$. In the following we verify the claim.

First let $n<u_{0}$. Then $k_{n}=0$. Therefore $n-k_{n}=n>r^{\alpha} \geq \alpha r$ and the claim is proved for this case. Second let $n=u_{0}$. Thus $k_{n}=1$ and $n-k_{n}=n-1 \geq r^{\alpha} \geq \alpha r$, so the claim is proved for this case too. Now let $n>u_{0}$. Since $n>r^{\alpha}$, we have $n>\alpha r$. We divide the proof into the following three cases. 
Case 1. $\alpha r<u_{0}<n$. Then $k_{n}=\left[\frac{n-u_{0}}{r+1}\right]+1$. So we have

$$
\alpha r+k_{n} \leq \alpha r+\frac{n-u_{0}}{r+1}+1 \leq u_{0}+\frac{n-u_{0}}{r+1}=\frac{r u_{0}+n}{r+1}<n .
$$

Thus $\alpha r<n-k_{n}$ as claimed.

Case 2. $u_{0} \leq \alpha r<2 \alpha r \leq n$. Then $\frac{n}{2} \geq \alpha r$. Since $\alpha \geq 2$ and $r \geq 2$, we have $n>4$. Hence

$$
k_{n} \leq \frac{n-u_{0}}{r+1}+1 \leq \frac{n-1}{r+1}+1 \leq \frac{n-1}{3}+1<\frac{n}{2} .
$$

It follows that $n-k_{n}>n-\frac{n}{2}=\frac{n}{2} \geq \alpha r$ as claimed.

Case 3. $u_{0} \leq \alpha r<n<2 \alpha r$. Note that $r^{\alpha}<n$. Then we have $r^{\alpha}<2 \alpha r$. But $\alpha \geq 2$ and $r \geq 2$, so we get that $r=\alpha=2$, or $r=2$ and $\alpha=3$, or $r=3$ and $\alpha=2$. Consider the following three subcases.

Subcase 3.1. $r=\alpha=2$. Then we have $u_{0} \leq 4<n<8$. Hence $n=5,6$ or 7 and $u_{0}=1$ or 3 since $u_{0}$ and $r$ are coprime. We need to exclude the exceptional case that $u_{0}=1$ and $n=5$ for which we have $n-k_{n}=3<4=\alpha r$. So we must have $u_{0}=3$ if $n=5$. For $u_{0}=3$ and $n=5$, we have

$$
n-k_{n}=5-\left[\frac{5-3}{3}\right]-1=4=\alpha r
$$

as claimed. For $n=6$ and 7 , we have

$$
n-k_{n} \geq 6-\left[\frac{6-1}{3}\right]-1=4=\alpha r
$$

and

$$
n-k_{n} \geq 7-\left[\frac{7-1}{3}\right]-1=4=\alpha r
$$

respectively. Therefore the claim is proved for this subcase.

Subcase 3.2. $r=2$ and $\alpha=3$. Then $u_{0}<8<n<12$. It follows that

$$
n-k_{n} \geq n-\frac{n-u_{0}}{r+1}-1=\frac{2 n+\left(u_{0}-3\right)}{3} \geq \frac{2}{3}(n-1) \geq \frac{16}{3}>5 .
$$

So we have found that $n-k_{n} \geq 6=\alpha r$. The claim is proved for this subcase.

Subcase 3.3. $r=3$ and $\alpha=2$. Then $u_{0}<9<n<12$. We deduce that

$$
k_{n} \leq \frac{n-u_{0}}{r+1}+1 \leq \frac{n-1}{4}+1=\frac{n+3}{4} \text {. }
$$

It follows that

$$
n-k_{n} \geq n-\frac{n+3}{4}=\frac{3}{4}(n-1) \geq \frac{27}{4}>6=\alpha r
$$

as claimed. The claim is proved for this subcase.

From the claim we deduce immediately that $r^{\alpha} \mid\left(n-k_{n}\right)$ !. Let $\left(n-k_{n}\right) !=r^{\alpha} B_{n}$ with $B_{n} \geq 1$ an integer. Since $\left(r, u_{0}\right)=1$, we have $\left(r^{\alpha}, u_{k_{n}} u_{k_{n}+1} \cdots u_{n}\right)=1$. Then letting $k=k_{n}$ in (2.1) gives us

$$
r^{\alpha} B_{n} L_{n, k_{n}}=A_{n, k_{n}} \cdot u_{k_{n}} u_{k_{n}+1} \cdots u_{n} .
$$

It then follows from (3.1) that $r^{\alpha} \mid A_{n, k_{n}}$. Hence $A_{n, k_{n}} \geq r^{\alpha}$. Thus by (2.1) and Lemma 2.2 we get

$$
L_{n, k_{n}} \geq r^{\alpha} C_{n, k_{n}} \geq u_{0} r^{\alpha}(r+1)^{n}
$$


Therefore the conclusion follows immediately and the proof of Theorem 1.1 is complete.

Remark 3.1. We must point out that the restrictive condition $n>r^{\alpha}$ is necessary. Otherwise, Theorem 1.1 may not be true. For instance, let $u_{0}=r=2, \alpha=3$ and $n=r^{\alpha}=8$. Then $L_{n}=\operatorname{lcm}\{2,4,6,8,10,12,14,16,18\}=5040$. On the other hand, $u_{0} r^{\alpha}(r+1)^{n}=104976$. Therefore $L_{n}<u_{0} r^{\alpha}(r+1)^{n}$.

\section{REFERENCES}

1. T. M. Apostol, Introduction to analytic number theory, Springer-Verlag, New York, 1976. MR.0434929(55:7892)

2. G. Bachman and T. Kessler, On divisibility properties of certain multinomial coefficients. II, J. Number Theory 106 (2004), 1-12. MR2029778 (2005a:11019)

3. B. Farhi, Minorations non triviales du plus petit commun multiple de certaines suites finies d'entiers, C.R. Acad. Sci. Paris, Ser. I 341 (2005), 469-474. MR2180812 (2006g:11006)

4. B. Farhi, Nontrivial lower bounds for the least common multiple of some finite sequences of integers, J. Number Theory 125 (2007), 393-411. MR2332595

5. B. Green and T. Tao, The primes contain arbitrarily long arithmetic progressions, Ann. of Math. (2) 167 (2008), 481-548.

6. D. Hanson, On the product of the primes, Canad. Math. Bull. 15 (1972), 33-37. MR0313179 $(47: 1734)$

7. G. H. Hardy and E. M. Wright, An introduction to the theory of numbers, fourth edition, Oxford University Press, London, 1960. MR0067125 (16:673c)

8. S. Hong and W. Feng, Lower bounds for the least common multiple of finite arithmetic progressions, C.R. Acad. Sci. Paris, Ser. I 343 (2006), 695-698. MR2284695 (2007h:11004)

9. S. Hong and R. Loewy, Asymptotic behavior of eigenvalues of greatest common divisor matrices, Glasgow Math. J. 46 (2004), 551-569. MR2094810 (2005f:11040)

10. K. Ireland and M. Rosen, A classical introduction to modern number theory, 2nd edition, GTM 84, Springer-Verlag, New York, 1990. MR1070716(92e:11001)

11. G. Myerson and J. Sander, What the least common multiple divides. II, J. Number Theory 61 (1996), 67-84. MR.1418320 (97k:11003)

12. M. Nair, On Chebyshev-type inequalities for primes, Amer. Math. Monthly 89 (1982), 126129. MR643279 (83f:10043)

Mathematical College, Sichuan University, Chengdu 610064, People's Republic of CHINA

E-mail address: s-f.hong@tom.com, hongsf02@yahoo.com, sfhong@scu.edu.cn

Mathematical College, Sichuan University, Chengdu 610064, People's Republic of CHINA

E-mail address: y.j.yang@tom.com 